\title{
Partially Ordered Sets in Crystallography
}

\author{
M Julian ${ }^{1}$ \\ ${ }^{1}$ Virginia Tech, Blacksburg, VA \\ erie@vt.edu
}

I have always enjoyed examining crystallography through the lens of abstract algebra. In college, I had a double major of pure mathematics and physics. This work is part of a chapter in my next book.

Partially ordered sets are an excellent device to explore the relations among both point groups and space groups. Figure 1 is a Hasse diagram or tree of the three-dimensional point groups [1]. (Unfortunately, the word order has two different meanings. In 'partially ordered sets', order refers to sequence, in the 'order of a point group' order means the number of elements in the point group.) In my talk, set elements and ordering relations are defined. Partially ordered sets have 'incomparable' elements. A totally ordered set is a chain. The two-dimensional point group trees are labeled with the general position and symbol stereographic projection symbols. In my talk, the duality principle is applied to three-dimensional point groups to explore the relationships among the centrosymmetric point groups and piezoelectricity. At the Curie temperature barium titinate goes from a strongly piezoelectric material to a nonpiezoelectric material. Isomorphism is demonstrated in three systems: first, between the three-dimensional monoclinic and orthorhombic crystal systems; second, between the tetragonal and hexagonal systems; and third, between the trigonal and cubic systems. Mappings are given. The cubic system is an example of mathematical lattice. The two-dimensional space group tree has the order of the associated point group plotted along the y-axis. This is necessary because the individual space groups themselves are infinite. In my talk, the seven rectangular space groups are illustrated. The three-dimensional Type I space group Hasse diagram for space group $2, \mathrm{P} \square \square$, is shown limited to its maximal subgroups and minimal supergroups. Finally, Type I trees are shown for the 13 monoclinic space groups.

\section{Reference}

[1] Julian, Maureen M. Foundations of Crystallography with Computer Applications, 2nd edition, CRC Press: New York (2015), ISBN: 978-1466552913. Figure 3.54 p.131.

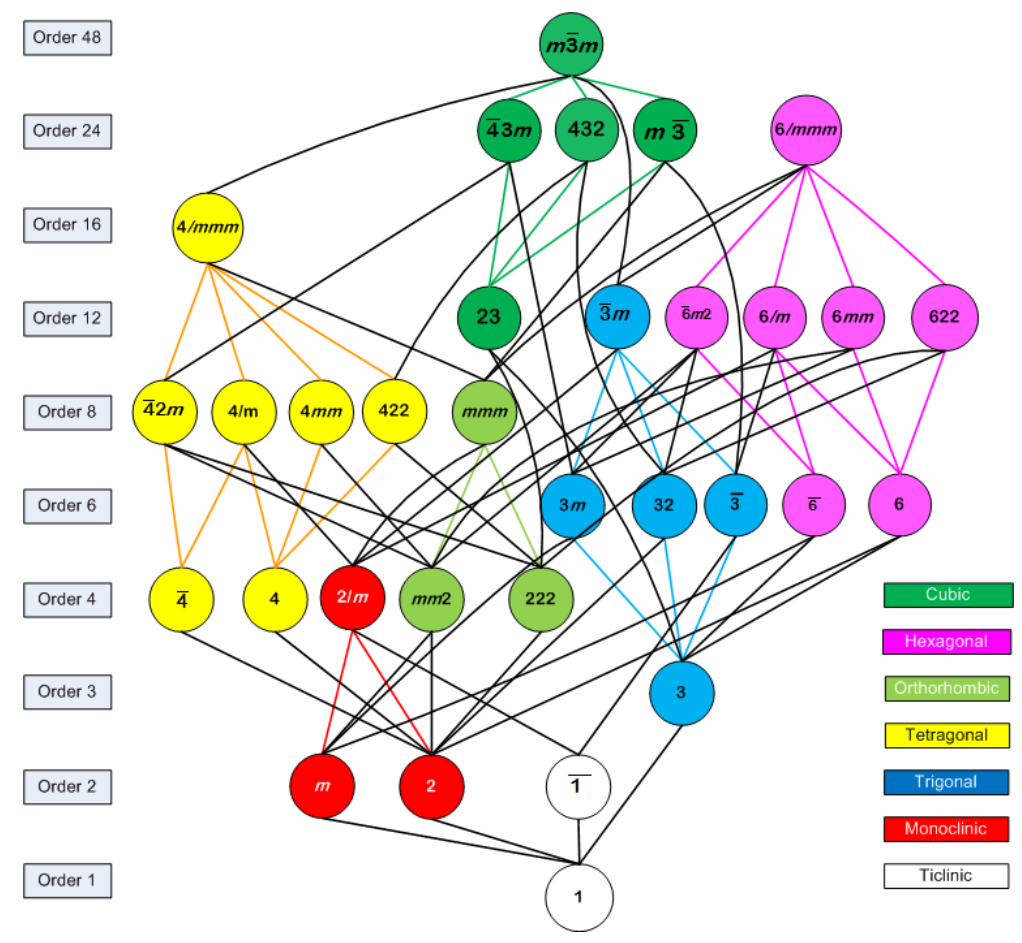

Figure 1. Hasse diagram of the three-dimensional point groups [1].

Acta Cryst. (2020). A76, a92 